\title{
Clinical Significance of Fever and Leukocytosis in Diagnosis of Acute Appendicitis in Children Who Visit Emergency Department With Abdominal Pain
}

\author{
Sang Hyun Haa , Chong Kun Hong ${ }^{\mathrm{a}}$, Younghwan Lee ${ }^{\mathrm{a}}$, Ae Jin Sunga ${ }^{\mathrm{a}}$, Jun Ho Lee ${ }^{\mathrm{a}}$, \\ Kwang Won Cho a, Seong Youn Hwang ${ }^{\mathrm{a}}$, Na Kyoung Lee ${ }^{\mathrm{a}}$, Hyeon Woo Yim ${ }^{\mathrm{b}, \mathrm{c}}$
}

\begin{abstract}
Background: The use of fever in the diagnosis of appendicitis in pediatric patients is unproven. The purpose of this investigation was to determine the value of fever in the diagnosis of acute appendicitis in children presenting to the emergency department (ED) with acute abdominal pain.
\end{abstract}

Methods: Medical records from January-December, 2009, were reviewed for children (age $<18$ years) who presented to an ED of one medical center with abdominal pain. Data on initial body temperature, white blood cell count, left shift, and final diagnosis were analyzed.

Results: Of 674 children, 119 had appendicitis. The prevalance of initial fever did not differ between those with and without appendicitis, but the prevalence of leukocytosis and left shift were higher in children diagnosed with appendicitis. Despite of stratification by age, leukocytosis, prevalence of fever was not differing.

Conclusions: Fever does not help in diagnosing appendicitis in our patients. Leukocytosis and white cell left shifts are on the other hand more common in patients with appendicitis. More studies should follow to investigate their role in diagnosis of appendicitis

Manuscript accepted for publication January 26, 2012

${ }^{a}$ Departments of Emeregncy Medicine and Nursing, Samsung Changwon Hospital, Sungkyunkwan University School of Medicine, Changwon 630-522, South Korea

${ }^{b}$ Department of Preventive Medicine, College of Medicine, The Catholic University of Korea, Seoul, South Korea

${ }^{\mathrm{c} C}$ Corresponding author: Hyeon Woo Yim, Department of Preventive Medicine, College of Medicine, The Catholic University of Korea, 505

Banpo-dong, Seocho-gu, Seoul, 137-701, South Korea.

Email: schsfc@hanmail.net

doi:10.4021/ijcp104e in patients in emergency department.

Keywords: Fever; White blood cell count; Appendicitis

\section{Introduction}

Acute abdominal pain is one of the most common complaints in patients who visit the emergency department (ED). When a patient complains of pain in the right lower quadrant of the abdomen, a clinician faces the challenge of considering acute appendicitis as a possibility [1]. In Korea and internationally, acute appendicitis is one of the most common causes of acute abdominal pain requiring surgical intervention [1]. In cases of delayed diagnosis or misdiagnosis, possible complications including abscess, peritonitis, sepsis, intestinal obstruction and, ultimately, death, may occur [2]. Early diagnosis and treatment of acute appendicitis is crucial. However, the diagnosis is still somewhat difficult from the clinician's point of view, especially for children. This is because acute appendicitis in children presents symptoms that are often similar to symptoms of illnesses, such as acute gastroenteritis, urinary tract infection, or fecal impaction. Furthermore, communication difficulties related to the young age of a pediatric patient can make a diagnosis more time-consuming and difficult, compared to adults [3]. A misdiagnosis rate of $28 \%-57 \%$ has been reported in children $\leq$ 12-years-of-age [3-7].

The Alvarado score for differential diagnosis of acute appendicitis by clinical characteristics and pediatric appendicitis score for pediatric patients have been reported and their diagnostic validities verified [8-11]. Fever and leukocytosis are included in both scoring systems, and several reports have indicated that the presence of fever is indicative of acute appendicitis $[6,12,13]$. However, in another study, in adults diagnosed with acute appendicitis, $15.6 \%$ had no fever or leukocytosis [14]. Furthermore, in a study of pediatric patients $\leq 6$-years-of-age who visited the ED with abdominal pain, no significant difference in the frequency of fever was evident between patients diagnosed with acute appendicitis and patients who were not [3]. 
We conducted this study to ascertain if the presence of fever and leukocytosis are indicative factors in the diagnosis of acute appendicitis in children who visit the ED with abdominal pain.

\section{Materials and Methods}

\section{Subjects}

This study used data from a regional emergency medical center visited by approximately 30,000 patients annually. We retrospectively investigated medical records of patients aged $\leq 18$-years-of-age who visited the ED from January 1 to December 31, 2009, with the initial chief symptom of abdominal pain (coded as C0000737, following questioning by the attending ED nurse). Because vague abdominal complaints predominates in children than adults, we intended to included all abdominal pain in addition to specific right quadrant pain. This study was approved by the hospital's Clinical Study Ethics Committee (IRB No. 2010- SCMC-020-00).

\section{Methods}

Patient records were excluded if the possibility of acute appendicitis had been ruled out, according to the following criteria: 1) past history of appendectomy, 2) abdominal pain occurring after trauma, 3) abdominal pain related to delivery, and 4) abdominal pain after foreign body ingestion. One investigator submitted the data in a pre-established computerized format. In cases of hospitalized patients, or in patients who visited the ED from an outpatient clinic, we also examined the admission records and outpatient clinic records, respectively. If the data from the ED, admission, and outpatient clinic records differed, the ED records were selected for analysis. Records from a total of 765 patients were initially enrolled. Exclusions were made based on the following: past history of appendectomy $(\mathrm{n}=18)$, abdominal pain occurring post-trauma $(\mathrm{n}=9)$, abdominal pain related to delivery $(\mathrm{n}=$ $2)$, abdominal pain after foreign body ingestion $(\mathrm{n}=1)$, and 12 patients for whom ED medical records were not made. To strengthen the accuracy of final diagnosis, we conducted telephone follow-ups with 329 patients who had not been hospitalized in our hospital or who did not have pathological examination results. We investigated any recurrence of symptom within 1 month after discharge from the ED or if other diagnoses had been made by different medical institutions. Further exclusions are made for 49 patients who either refused to answer the phone survey questions or could not be contacted by calling twice within a 3-day interval. One hundred nineteen patients had a diagnosis of acute appendicitis in their medical records (appendicitis group) and, with the exclusion of aforementioned patients, 329 patients were not diagnosed with appendicitis (Fig. 1). While acute appendicitis has been treated using only antibiotics [15], we assumed that acute appendicitis causes pain in the majority of cases and that the situation cannot be healed naturally. We conducted this study using data from 674 patients and investigated age, sex, initial body temperature, white blood cell (WBC) count, discharge diagnosis recorded in ED or in the ward, pathological examination results, abdomen computed tomography (CT) or ultrasound results. Blood tests are done using a model XE-2100 apparatus (Sysmex, Kobe, Japan) and body temperature was measured at the axilla using a model MT 200 thermometer (Microlife, Basel, Switzerland) during the period of study in our ED.

\section{Definitions of study variables and outcomes}

Acute appendicitis group refers to patients with confirmed acute appendicitis by pathological examination of the specimen. Mesenteric lymphadenitis group refers to patients without evidence of acute appendicitis as detected by abdominal CT or abdominal ultrasound, other than enlargement of mesenteric lymph nodes. Acute gastroenteritis group refers to patients with a recorded final diagnosis by the physicianin-charge and no relapse after discharge and no other diagnosis made by different, or the same medical institution, as revealed in telephone follow ups. Fecal impaction group refers to patients with a recorded final diagnosis by the physician-in-charge, with improvement of symptoms after enema, as recorded in a nursing chart, and no relapse after discharge or other diagnosis made by different, or the same medical institution, as revealed in telephone follow ups. Other groups refer to patients not included in the previous categories. Non-appendicitis group refers to patients in the mesenteric lymphadenitis, acute gastroenteritis, fecal impaction, and other group categories. Body temperature refers to axillary temperature measured and recorded by the ED nurse upon patient presentation at the ED. Fever refers to a body temperature above 37.4 oC [16]. WBC count refers to the first WBC count measured after entering the ED. Shift of $\mathrm{WBC}$ to the left (or left shift) refers to the ratio of segmented neutrophil and band form $75 \%$ or above, in the first WBC count measured after entering the ED.

\section{Analyses}

For final diagnosis, we used pathological diagnosis if a patient had an operation in our hospital and, thus, had a pathological examination of the removed specimen. In other cases, we used the diagnosis made by the attending doctors when the patients were discharged from an ED or a ward. For patients discharged from an ED, we used an ED discharge diagnosis as the final diagnosis if symptoms did not recur or no different diagnosis made by other medical institution, as checked by our telephone follow-ups. We divided 674 patients into the acute appendicitis and non-acute appendicitis 


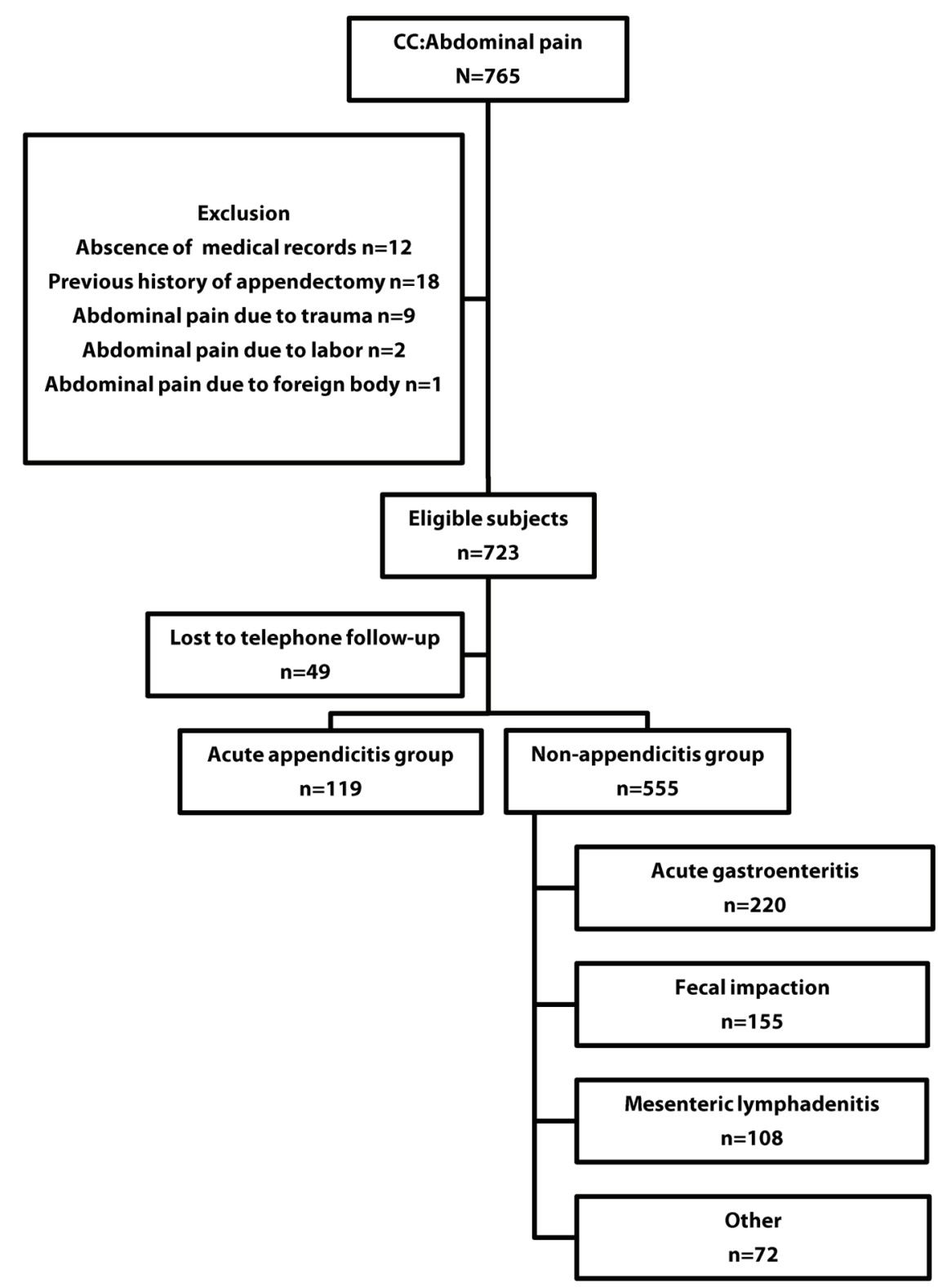

Figure 1. Flow chart of patients with appendicitis.

groups, and compared the frequency of fever as an initial presenting symptom. Because of the difference between the body temperature and rectal temperature, we used $37.4 \mathrm{oC}$ or higher, to make it comparable to fevers in other studies that defined fever as body temperature $\geq 38$ oC [16]. Also, we compared the frequency of leukocytosis between the groups using a WBC standard of $>10,000$ cells $/ \mathrm{mm}$, consistent with most studies [3, 17-20]. Lastly, to compensate for the effect of change in leukocyte levels, shift of WBC to the left and age on the temperature, we stratified each of these factors and used receiver operating characteristic (ROC) curve analysis to analyze diagnostic significance of fever $(\geq 37.4$ oC). Statistical analysis was performed by PASW ver. 18.0 (SPSS, Chicago, IL). Two sample T-test was used for con- tinuous data and was converted into categorical data when needed, followed by Chi-squared test. Statistical significance was defined at $\mathrm{P}<0.05$.

\section{Results}

\section{Characteristics of the study population}

Seven hundred and sixty five patients visited our emergency center during the 1-year period of data collection. Data from 674 patients was used. One hundred nineteen patients were diagnosed with acute appendicitis, and 555 of them were not (Fig. 1). We were able to acquire WBC level data in all 119 
Table 1. Comparison of Characteristics of Study Population

\begin{tabular}{|c|c|c|c|}
\hline Characteristics & $\begin{array}{l}\text { Appendicitis group } \\
(n=119)\end{array}$ & $\begin{array}{l}\text { Non appendicitis group } \\
(\mathrm{n}=\mathbf{5 5 5})\end{array}$ & P value \\
\hline Age (years) & $11.5 \pm 4.2$ & $8.6 \pm 5.3$ & $<0.001^{*}$ \\
\hline Toddler ( 1 - 3.9 years $)$ & $2(1.7 \%)$ & $125(22.5 \%)$ & 0.324 \\
\hline Child (4 - 11.9 years) & $58(48.7 \%)$ & $238(42.9 \%)$ & 0.129 \\
\hline Adolescent (12 - 18 years) & $59(49.6 \%)$ & $192(34.6 \%)$ & 0.210 \\
\hline Male sex $(\%)$ & $59.7 \%$ & $53.7 \%$ & 0.235 \\
\hline Initial temperature $\left({ }^{\circ} \mathrm{C}\right.$, axillary) & $36.8 \pm 0.6$ & $36.6 \pm 0.7$ & 0.188 \\
\hline WBC count $\left(\times 1000 / \mathrm{mm}^{3}\right)$ & $14.8 \pm 4.8$ & $9.7 \pm 4.1$ & $<0.001^{*}$ \\
\hline Segment neutrophil (\%) & $78.6 \pm 12.4$ & $64.4 \pm 16.4$ & $<0.001^{*}$ \\
\hline
\end{tabular}

*: statistical significance was established at a level of $P<0.05$.

patients from the acute appendicitis group and 398 of 555 patients in the non-acute appendicitis group.

No difference in sex was found between the groups. However, the acute appendicitis group had more aged patients. Initial body temperatures first measured after entering an ED did not differ significantly between the groups. Fever $(\geq 37.4 \mathrm{oC}$ ) was evident in $11.8 \%$ and $13.7 \%$ of patients in the acute appendicitis and non-appendicitis group, respectively, and the difference was not significant (Table 1). We categorized the patients into three age groups: $1-3,4$ 11 , and $12-18$ years. No age-related significant differences were evident (Table 2).

WBC levels obtained from 517 of the 674 patients were analyzed using two samples T-test. The WBC level in the acute appendicitis group $\left(14,800 / \mathrm{mm}^{3}\right)$ was significantly higher than in the non-appendicitis group $\left(9,700 / \mathrm{mm}^{3}\right)$. Defining WBC level $\geq 10,000 / \mathrm{mm}^{3}$ as leukocytosis, results of $\chi^{2}$ test showed a significantly higher frequency of leukocytosis in the acute appendicitis group (Table 1). In the age-stratified analysis, all patients in the acute appendicitis group showed significantly high frequency of leukocytosis, except for $\leq$ 3-years-of-age (Table 2).

The segment neutrophil ratio in both groups was analyzed using two samples T-test; the $78.6 \%$ of the acute appendicitis group was significantly higher than the $64.4 \%$ of the non-appendicitis group (Table 1).

Defining the left shift of $\mathrm{WBC}$ as $\geq 75 \%$, the results of $\chi^{2}$ test showed significantly high frequency of the left shift of WBC in the acute appendicitis group (Table 1). In the agestratified analysis, all in the acute appendicitis group showed significantly high frequency of the left shift of WBC, except for patients $\leq 3$-years-of-age (Table 2 ).

We categorized the patients in the non-appendicitis group into four subgroups, according to their final diagnosis (i.e., acute gastroenteritis group, fecal impaction group, mesenteric lymphadenitis group, and other). Other included abdominal pain due to ovarian cyst $(\mathrm{n}=8)$, urolithiasis ( $\mathrm{n}$ $=4)$, nonspecific abdominal pain $(\mathrm{n}=5)$, and acute upper respiratory tract infection $(n=8)$. The fecal impaction group had significantly lower age. No significant differences in sex and initial body temperature were found in the four groups. WBC level and segment neutrophil ratio were significantly high in patients in the 'other' group (Table 3).

Significance of initial body temperature, WBC level, and left shift of WBC on diagnosis of acute appendicitis, and ROC curve analysis

Initial body temperature, WBC level, and left shift of WBC were analyzed using ROC curve to find out their diagnostic significance. Initial body temperature was insignificant in the diagnosis of acute appendicitis (Fig. 2A). However, the WBC level (Fig. 2B) and the segment neutrophil ratio (Fig. 2C) showed an area-under the curve value of 0.815 and 0.766 , respectively, implying a diagnostic value.

Significance of initial body temperature on diagnosis of acute appendicitis: stratified ROC curve analysis

The significance of initial body temperature on diagnosis of 


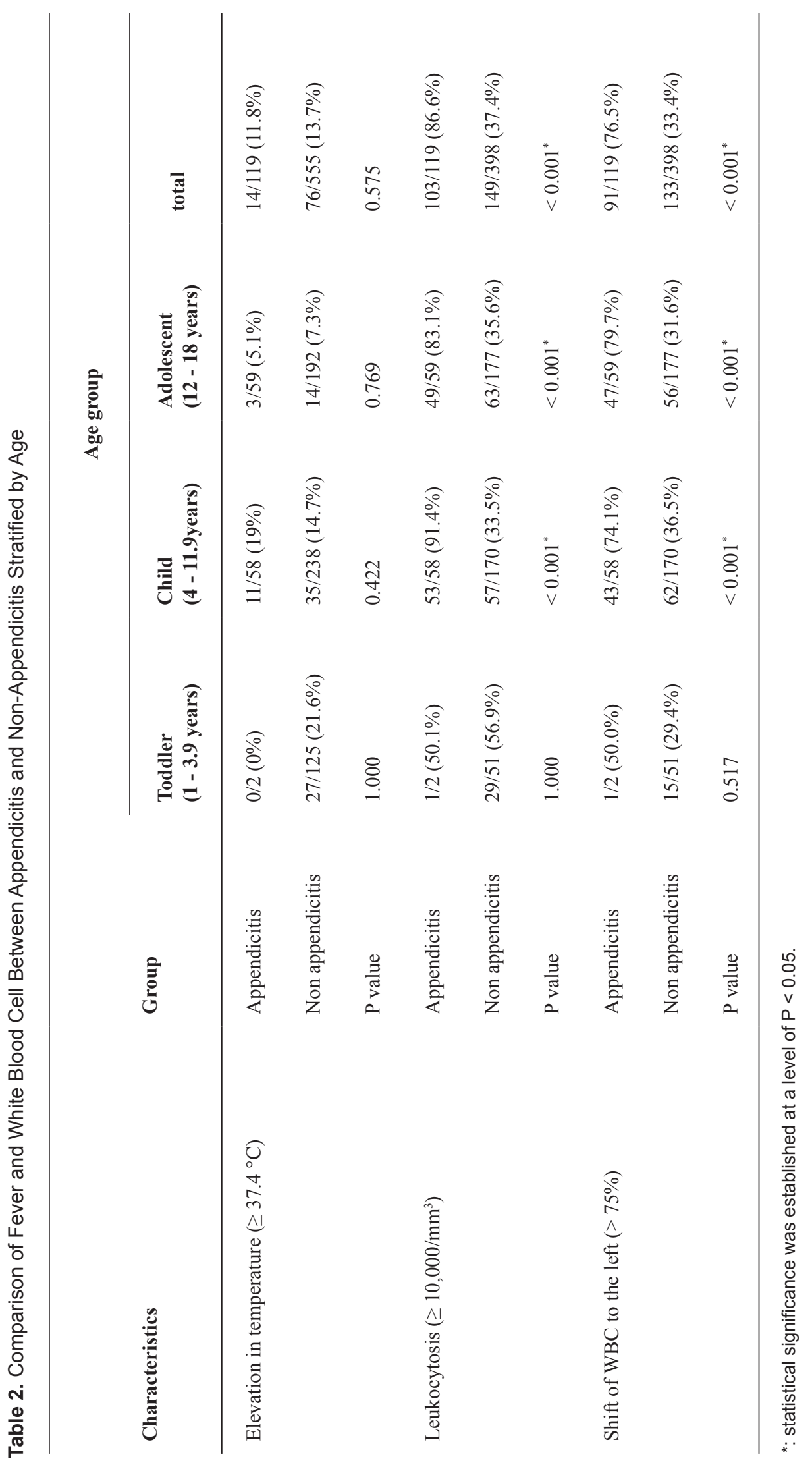


acute appendicitis was analyzed using ROC curve and using the following stratified factors: WBC level of 10,000/ $\mathrm{mm}^{3}$ [17-20] (and higher or lower), segment neutrophil ratio of $75 \%$ (and higher or lower), and age. Patients with WBC level $\geq 10,000 / \mathrm{mm}^{3}$ were analyzed using ROC curve; the results indicated that body temperature had no diagnostic value. Similar results were found when the patients with WBC level $<10,000 / \mathrm{mm}^{3}$ were analyzed using ROC curve (Fig. $3 \mathrm{~A}, \mathrm{~B})$. In analyses of stratified factors of segment neutrophil ratio, no diagnostic value of body temperature was evident (Fig. 3C, D). In the age-stratified analysis, all three groups showed no diagnostic value of body temperature (Fig. 3E, F, G). When we specifically extracted the mesenteric lymphadenitis group (mesenteric lymphadenitis is an illness that is difficult to differentiate from acute appendicitis), and compared it to the acute appendicitis group, body temperature was not useful in differentiating the two illnesses (Fig. 3H).

\section{Discussion}

Early diagnosis of acute appendicitis in pediatric patients remains difficult, even for an experienced clinician. The diagnostic approaches of two scoring systems $[10,21]$ have been verified as being useful in diagnosis $[8,9]$. However, the diagnostic value of fever or leukocytosis is debatable [3, $6,12-14,21]$. Monneuse et al. analyzed 326 patients suspected of acute appendicitis prior to surgical intervention and reported that fever or leukocytosis was not detected in $15.6 \%$ of the patients [14]. The authors suggested that fever and leukocytosis cannot be reliably used to rule out acute appendicitis. Coleman et al. categorized acute appendicitis patients into four groups (acute, necrotic, abscess-forming, and peritonitis-forming) and reported no difference in frequency of leukocytosis [21]. In addition, the authors categorized all the acute appendicitis patients into a leukocytosis group and non-leukocytosis group; no difference in body temperature between the groups was found.

In contrast, Wang et al. studied patients who visited the ED with a chief complaint of abdominal pain, and reported that in cases of co-existing leukocytosis and left shift of WBC, the sensitivity and specificity of diagnosis of acute appendicitis was $79 \%$ and $80 \%$, respectively, evidence that leukocytosis is an indicative finding of acute appendicitis[22].

In this study, we retrospectively investigated medical records of 765 patients aged $\leq 18$-years-of-age who visited one emergency center during a 1-year period with initial chief symptom of abdominal pain, with the aim of clarifying the diagnostic value of fever in acute appendicitis. We compared 119 patients with a final diagnosis of acute appendicitis with 555 patients not diagnosed with the illness.

The age of the acute appendicitis group was higher than the non-acute appendicitis group. We assume this is because we included children of all pediatric ages, from $1-18$ 

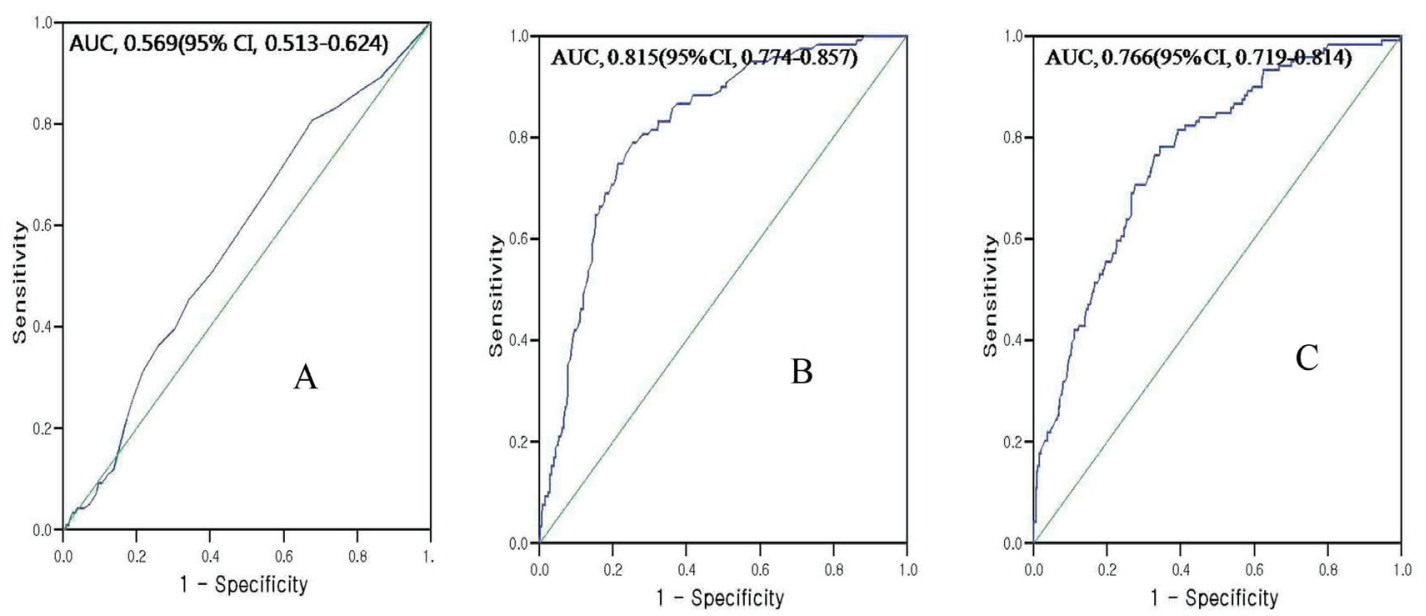

Figure 2. ROC points and fitted AUCs for the acute appendicitis. ROC points and fitted AUCs for fever for the acute appendicitis (A), ROC points and fitted AUCs for WBC count for the acute appendicitis (B), ROC points and fitted AUCs for segment neutrophil for the acute appendicitis (C).

years, and the fact that the frequency of acute appendicitis is relatively low in children aged $\leq 3$ years. Among the 119 acute appendicitis patients, only two were $\leq 3$-years-of-age (1.6\%), similar to result reported by Alloo et al.[6].

In this study, the definition of fever obtained by axillary measurement, consistent with the norm, was $\geq 37.4 \mathrm{oC}[17$, 20]. Lin et al. reported that an acute appendicitis group had higher frequency of fever in comparison to a non-appendicitis group ( $43.4 \%$ and $17 \%$, respectively) in a study of pediatric patients who visited the ED with abdominal pain in the right lower quadrant [13]. However, in this study, the average body temperature in the acute appendicitis group and non-appendicitis group was $36.8 \mathrm{oC}$ and $36.6 \mathrm{oC}$, respectively, which was statistically insignificant. Colvin et al. also reported similar results, in which body temperatures in both groups were the same, at $37.4 \mathrm{oC}$ at the time of admission to the $\mathrm{ED}$, similar to the assertion that the initial body temperature does not aid in differential diagnosis of acute appendicitis [3]. When the acute appendicitis and non-appendicitis patients were grouped into two according to presence or absence of fever, then compared with each other, the frequencies of fever were $11.8 \%$ and $13.7 \%$, respectively, and the difference was not significant. A similar result was found in the age-stratified analysis of the groups. This is similar to the report of Colvin et al., in which an age-stratified analysis of the group withfever $\geq(38 \mathrm{oC})$ and the group which had not, and reported no difference in all the age groups [3].

The majority of studies $[6,13,22,23]$ assert that an increase in WBC level indicates acute appendicitis. A similar result was found in our study. In the acute appendicitis group, the average WBC level was $14,800 / \mathrm{mm}^{3}$, which was higher than the $9,700 / \mathrm{mm}^{3}$ of the non-acute appendicitis group. The segment neutrophil ratio was also higher in the acute appendicitis group. In age-stratified analysis, only the age group of $\leq 3$ years did not show difference in frequency of leukocytosis and frequency of the left shift of WBC. But, only two patients were aged under 3 in our study, making if imprudent to generalized our finding. However, it is relevant that Paajanen et al. also reported a weak inflammatory reaction in response to acute appendicitis in infants and, hence, a feeble increase in WBC level [24].

To investigate the diagnostic value of body temperature and to eliminate confusion derived from factors such as WBC level, we stratified each factor then conducted ROC curve analysis, which showed insignificance in most cases. In the 4 - 11 year-old age group, however, the analysis did reveal the diagnostic significance of fever. But, when the area under the curve of 0.652 was applied alone, its diagnostic value seemed feeble.

When we specifically compared the mesenteric lymphadenitis group (a non-acute appendicitis group) to the acute appendicitis group, the results showed that body temperature was not helpful in differentiating the two illnesses.

Characteristics, as well as limitations, of this study are as follows. Firstly, we investigated all patients who visited a single hospital with abdominal pain, hence there was no selection bias. The percentage of patients diagnosed with acute appendicitis among those who came to the hospital with abdominal pain was $17.7 \%$, which is considered high. This is because, as a tertiary medical institution, our hospital has many referrals of patients suspected of having acute appendicitis from primary and secondary medical institutions, which explains the higher frequency of patients requiring surgical intervention among those who came to the hospital with abdominal pain. As such, the results from this study may not be applicable to the circumstances in primary and secondary medical institutions. Among the 119 patients, there were only two patients between the ages of 1 and 3.9 years who were analyzed. It is well-known that fewer than $5 \%$ of patients diagnosed with appendicitis are $\leq 4$-years-of- 

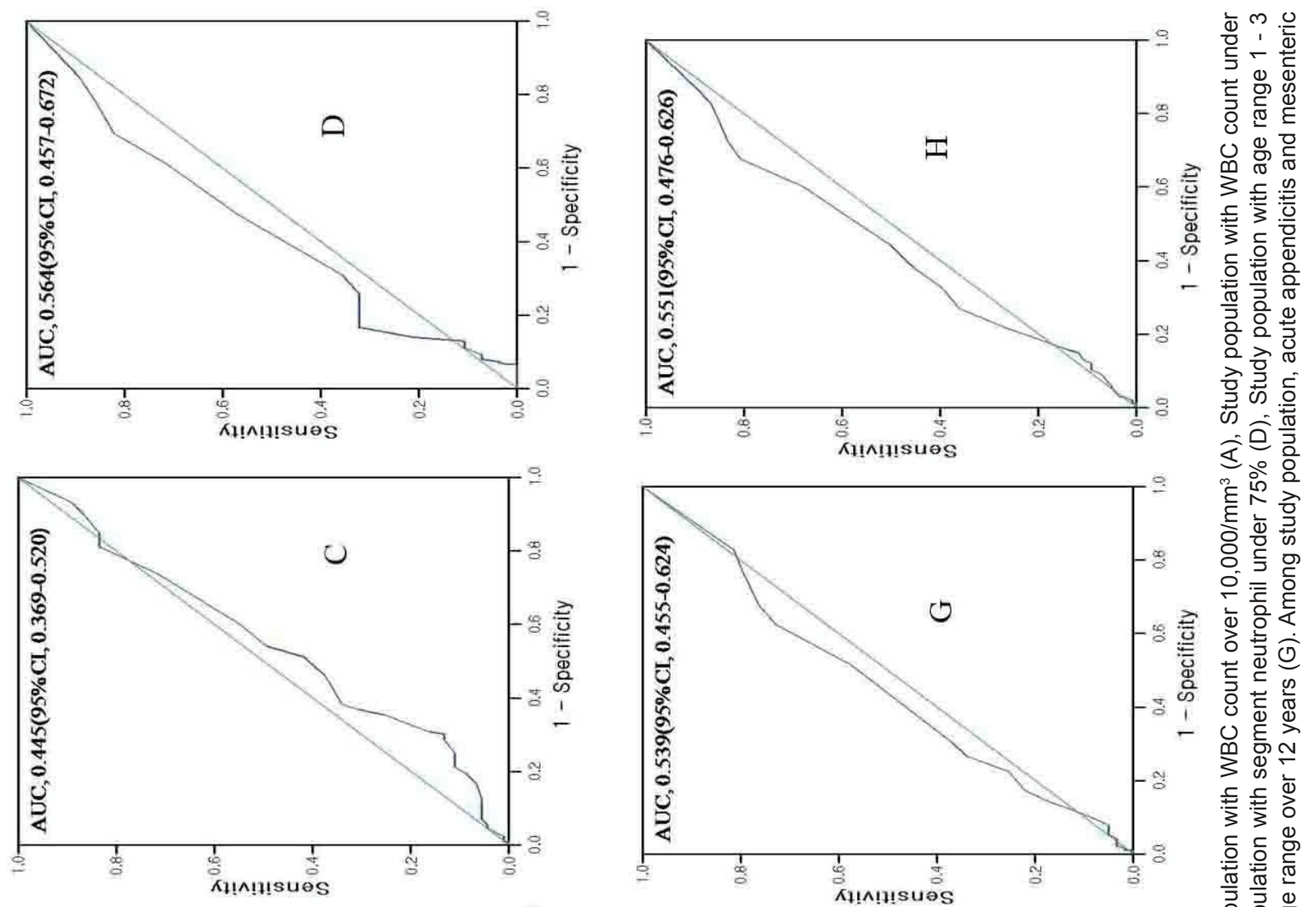

$\stackrel{2}{\wedge} \frac{1}{x}$

है 흘

응 인

을 등 흥

号守
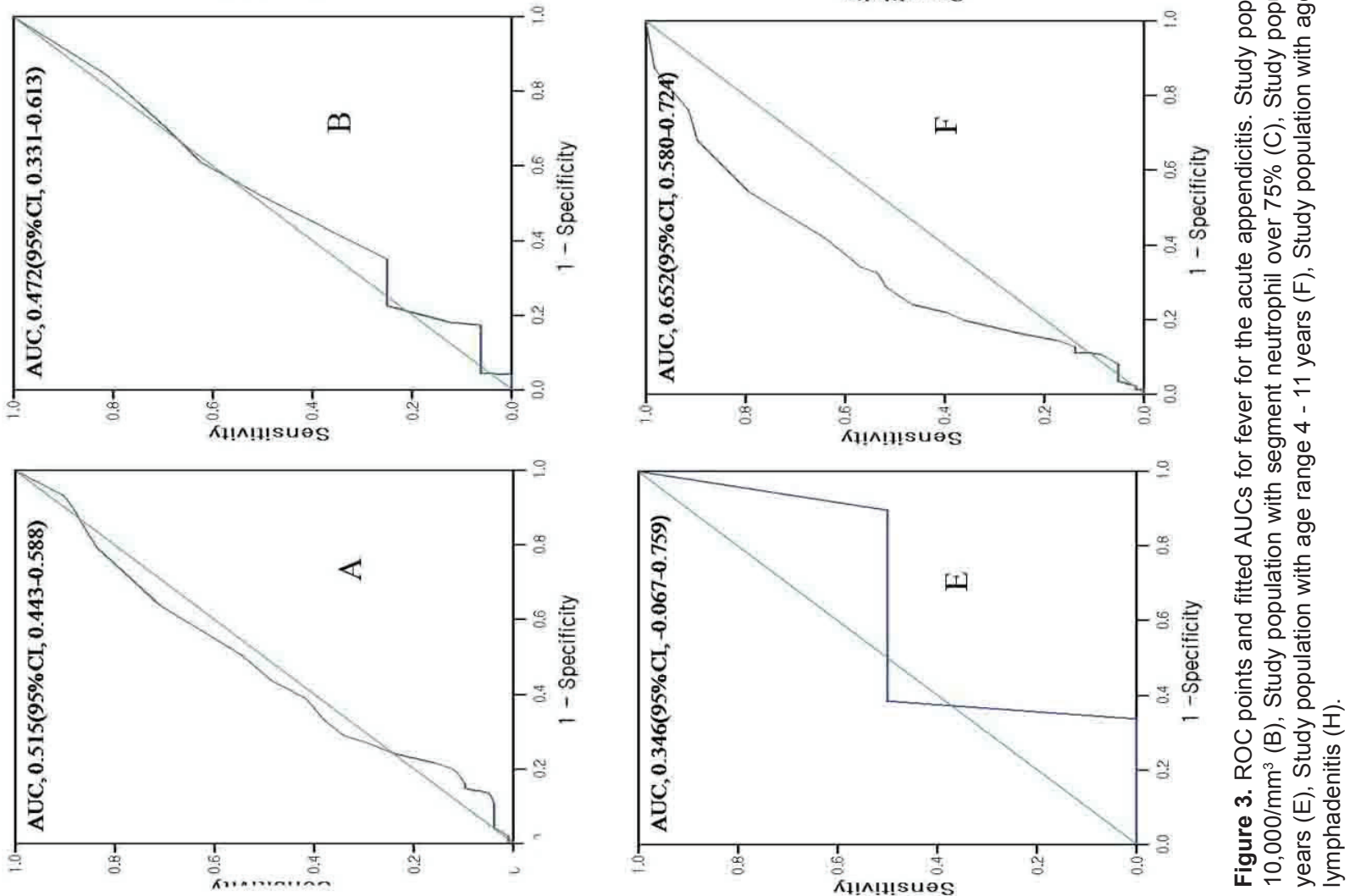
age. Our results are consistent with these findings, but the small number of patients in this age group makes any conclusions from the present data impossible. For this reason, a long-term, prospective, observational cohort study is needed.

Secondly, this study was a retrospective, cross-sectional study that was based on medical records. Hence, the possibility of measurement error and erroneous data may exist, and there is the possibility that bias may have intervened during data collection. To minimized bias in this study, we only used body temperature and WBC level, which are objective indices. To strengthen the final diagnosis of patients with abdominal pain, we conducted telephone follow-ups with those who had been discharged from the ED, in an attempt to minimize measurement error.

Thirdly, as one of limitations of a retrospective, crosssectional study, administration of an antipyretic prior to visiting ED was not recorded in the charts, so we could not check any possible effect of antipyretic administration. However, in our study, the initial body temperature was not diagnostically important in diagnosis of acute appendicitis in patients found in emergency settings.

In conclusion, in patients $\leq 18$-years-of-age who visited the ED with the chief complaint of abdominal pain, no difference in frequency of fever was found between the acute appendicitis group and non-acute appendicitis group. Fever does not help in diagnosing appendicitis in our patients. But leukocytosis and white cell left shifts are on the other hand more common in patients with appendicitis. More studies should follow to investigate their role in diagnosis of appendicitis in patients in emergency department.

\section{Acknowledgment}

There is no financial support from any institutes and the authors individually or collectively have no significant financial conflicts that need disclosing.

\section{Conflict of Interest Statement}

The authors have no conflict of interests and no financial disclosures.

\section{References}

1. Lee JH, Park YS, Choi JS. The epidemiology of appendicitis and appendectomy in South Korea: national registry data. J Epidemiol. 2010;20(2):97-105.

2. Rothrock SG, Pagane J. Acute appendicitis in children: emergency department diagnosis and management. Ann Emerg Med. 2000;36(1):39-51.

3. Colvin JM, Bachur R, Kharbanda A. The presentation of appendicitis in preadolescent children. Pediatr Emerg Care. 2007;23(12):849-855.

4. Rothrock SG, Skeoch G, Rush JJ, Johnson NE. Clinical features of misdiagnosed appendicitis in children. Ann Emerg Med. 1991;20(1):45-50.

5. Nance ML, Adamson WT, Hedrick HL. Appendicitis in the young child: a continuing diagnostic challenge. Pediatr Emerg Care. 2000;16(3):160-162.

6. Alloo J, Gerstle T, Shilyansky J, Ein SH. Appendicitis in children less than 3 years of age: a 28 -year review. Pediatr Surg Int. 2004;19(12):777-779.

7. Paajanen H, Somppi E. Early childhood appendicitis is still a difficult diagnosis. Acta Paediatr. 1996;85(4):459462.

8. Goldman RD, Carter S, Stephens D, Antoon R, Mounstephen W, Langer JC. Prospective validation of the pediatric appendicitis score. J Pediatr. 2008;153(2):278282.

9. Bhatt M, Joseph L, Ducharme FM, Dougherty G, McGillivray D. Prospective validation of the pediatric appendicitis score in a Canadian pediatric emergency department. Acad Emerg Med. 2009;16(7):591-596.

10. Alvarado A. A practical score for the early diagnosis of acute appendicitis. Ann Emerg Med. 1986;15(5):557564.

11. Kharbanda AB, Taylor GA, Fishman SJ, Bachur RG. A clinical decision rule to identify children at low risk for appendicitis. Pediatrics. 2005;116(3):709-716.

12. Graham JM, Pokorny WJ, Harberg FJ. Acute appendicitis in preschool age children. Am J Surg. 1980;139(2):247250.

13. Lin CH, Chen JH, Li TC, Ho YJ, Lin WC. Children presenting at the emergency department with right lower quadrant pain. Kaohsiung J Med Sci. 2009;25(1):1-9.

14. Monneuse O, Abdalla S, Pilleul F, Hervieu V, Gruner L, Tissot E, Barth X. Pain as the only consistent sign of acute appendicitis: lack of inflammatory signs does not exclude the diagnosis. World J Surg. 2010;34(2):210215.

15. Mason RJ. Surgery for appendicitis: is it necessary? Surg Infect (Larchmt). 2008;9(4):481-488.

16. Temperature measurement in paediatrics. Paediatr Child Health. 2000;5(5):273-284.

17. Pearl RH, Hale DA, Molloy M, Schutt DC, Jaques DP. Pediatric appendectomy. J Pediatr Surg. 1995;30(2):173178; discussion 178-181.

18. Wu HP, Chang CF, Lin CY. Predictive inflammatory parameters in the diagnosis of acute appendicitis in children. Acta Paediatr Taiwan. 2003;44(4):227-231.

19. Harland RN. Diagnosis of appendicitis in childhood. J R Coll Surg Edinb. 1991;36(2):89-90.

20. van den Broek WT, van der Ende ED, Bijnen AB, Breslau PJ, Gouma DJ. Which children could benefit from additional diagnostic tools in case of suspected appendi- 
citis? J Pediatr Surg. 2004;39(4):570-574.

21. Coleman C, Thompson JE, Jr., Bennion RS, Schmit PJ. White blood cell count is a poor predictor of severity of disease in the diagnosis of appendicitis. Am Surg. 1998;64(10):983-985.

22. Wang LT, Prentiss KA, Simon JZ, Doody DP, Ryan DP. The use of white blood cell count and left shift in the diagnosis of appendicitis in children. Pediatr Emerg Care.
2007;23(2):69-76

23. $\mathrm{Ng} \mathrm{KC,} \mathrm{Lai} \mathrm{SW.} \mathrm{Clinical} \mathrm{analysis} \mathrm{of} \mathrm{the} \mathrm{related} \mathrm{factors}$ in acute appendicitis. Yale J Biol Med. 2002;75(1):4145.

24. Paajanen H, Mansikka A, Laato M, Kettunen J, Kostiainen $\mathrm{S}$. Are serum inflammatory markers age dependent in acute appendicitis? J Am Coll Surg. 1997;184(3):303308. 